Behavioral/Cognitive

\title{
Spatially Distinct Beta-Band Activities Reflect Implicit Sensorimotor Adaptation and Explicit Re-aiming Strategy
}

\author{
Amirhossein Jahani, ${ }^{1}$ Antoine Schwey, ${ }^{1}$ Pierre-Michel Bernier, ${ }^{2,3}$ and Nicole Malfait ${ }^{1}$ \\ ${ }^{1}$ Institut de Neurosciences de la Timone, Unité Mixte de Recherche 7289, Centre National de la Recherche Scientifique/Aix Marseille Université, 13385 \\ Marseille Cedex 5, France, ${ }^{2}$ Département de Kinanthropologie, Faculté des Sciences de l'activité Physique, Université de Sherbrooke, Sherbrooke, Québec \\ J1H 5N4, Canada, and ${ }^{3}$ Centre de Recherche du CHUS, Université de Sherbrooke, Sherbrooke, Québec J1H 5N4, Canada
}

Previous research suggests that so-called implicit and explicit processes of motor adaptation are implemented by distinct neural structures. Here we tested whether implicit sensorimotor adaptation and strategic re-aiming used to reduce movement error are reflected by spatially distinct EEG oscillatory components. We analyzed beta-band oscillations $(\sim 13-30 \mathrm{~Hz})$, which have long been linked to sensorimotor functions, at the time when these adaptive processes intervene for movement planning. We hypothesized that beta-band activity within sensorimotor regions relates to implicit adaptive processes, whereas beta-band activity within medial motor areas reflects deliberate re-aiming. In female and male human volunteers, we recorded EEG in a motor adaptation task in which a visual rotation was introduced in short series of trials separated by unperturbed trials. Participants were instructed in advance about the nature of the visual perturbation and trained to counter it by strategically re-aiming at a neighboring target. Consistent with our hypothesis, we found that preparatory beta-band activities within the two regions exhibited different patterns of modulation. Beta power in lateral central regions was attenuated when a change in the visual condition rendered internal-model predictions uncertain. In contrast, beta power in medial frontal regions was selectively decreased when participants strategically re-aimed their reaches. We propose that the reduction in lateral central beta power reflects an increased weighting of peripheral sensory information implicitly triggered when an adaptive change in the sensorimotor mapping is required, whereas the reduction in medial frontal beta-band activity relates to the inhibition of automatic motor responses in favor of cognitively controlled movements.

Key words: beta-band oscillations; EEG; explicit strategy; implicit process; motor learning; reaching

Significance Statement

Behavioral and modeling studies have proposed that so-called implicit and explicit components of motor adaptation recruit different neural circuits. Here, we investigated whether these different processes are reflected by spatially distinct beta-band activities. Analyzing EEG signals at the time they influence movement planning, during the foreperiod, we found that beta power within lateral central regions was decreased when a change in visual conditions required implicit sensorimotor remapping, which may reflect enhanced sensory processing when internal-model predictions are rendered uncertain. In contrast, beta-band power within medial frontal areas was selectively attenuated when participants deliberately re-aimed their movements to improve task performance, which may be associated with the inhibition of automatic motor responses in favor of cognitively controlled movements.

\section{Introduction}

Since von Helmholtz's (1867) seminal work on prism adaptation, the idea that (at least) two qualitatively distinct processes contrib-

\footnotetext{
Received Aug. 1, 2019; revised Jan. 5, 2020; accepted Jan. 19, 2020.

Author contributions: A.J. and N.M. designed research; A.J. performed research; A.J., A.S., P.-M.B., and N.M. analyzed data; A.J. and A.S. edited the paper; P.-M.B. and N.M. wrote the paper.

This work was supported by European Union H2020 MSCA-ITN/ETN (GA 642961 PACE) program and Agence Nationale de la Recherche (ANR-18-CE37-0018-01). We thank Julie Alayrangues and Flavie Torrecillos for participating in pilot data recording.

The authors declare no competing financial interests.

Correspondence should be addressed to Nicole Malfait at nicole.malfait@univ-amu.fr or nmalfait@gmail.com.
}

ute to motor adaptation has been proposed by numerous authors (Malfait and Ostry, 2004; Redding and Wallace, 1996; Taylor et al., 2014). The initial response would be a cognitive strategy allowing rapid reduction of movement error. In the case of prismatic displacement, one may use error information from a previous target-pointing trial to deliberately change the aiming direction of a subsequent movement to reduce performance error. The second kind of response would be the implicit and slow 
developing sensorimotor remapping that represents "true sensorimotor adaptation.” Behavioral and modeling studies (Mazzoni and Krakauer, 2006; Taylor and Ivry, 2011) have suggested that these two components of motor learning are driven by different teaching signals and implemented by distinct neural circuits. There is good evidence that implicit sensorimotor remapping is driven by sensory prediction errors (Jordan and Rumelhart, 1992) and involves sensorimotor cortico-cerebellar loops (Doyon et al., 2003; Shadmehr and Krakauer, 2008). In contrast, the neurophysiology of cognitive strategies, guided by performance outcome, has been less investigated. Still, one candidate is the medial frontal cortex, conceived as the fulcrum of the cognitive control system, and known to be central in "higher-order" aspects of motor control, including goal representation and movement planning (Picard and Strick, 1996; Nakamura et al., 1998; Rushworth et al., 2004; Nachev et al., 2008).

Here, we analyzed beta-band oscillations ( $\sim 13-30 \mathrm{~Hz})$, which have long been linked to sensorimotor preparation functions, at the time when adaptive mechanisms intervene in selecting and shaping the forthcoming movement. From the current literature, a clear functional role of movement-related beta activity, within and beyond sensorimotor cortex, cannot be determined. Nevertheless, evidence suggests that, during early delay periods, it reflects the conjunction of multiple factors associated with sensory and cognitive aspects of motor control rather than pure motor processes per se (Baker, 2007; Engel and Fries, 2010; Jones et al., 2010; Saleh et al., 2010; van Ede et al., 2011; Jenkinson and Brown, 2011; Kilavik et al., 2013).

Our goal was to determine whether functionally and spatially distinct beta-band activities relate to implicit and explicit motor learning components. Concerning implicit learning, it has been shown that, during the foreperiod before movement onset, betaband activity within lateral central regions is modulated when sensorimotor adaptive mechanisms are automatically activated by movement-execution errors (Torrecillos et al., 2015; Alayrangues et al., 2019). In these studies, visual and/or mechanical perturbations were unexpectedly applied in isolated catch-trials, which induced slight involuntary movement deviations in the opposite direction on the following trial. As for the neural basis of flexible and deliberate behavioral adjustments, monkey work has highlighted the critical role of medial motor structures when controlled actions need to overcome inefficient automatic motor responses (Isoda and Hikosaka, 2007). In particular, analysis of local field potentials in the supplementary motor complex have revealed reduced beta power in this region during the early delay period when monkeys have to rapidly update a motor plan in response to a visual instruction (Hosaka et al., 2016).

In light of these findings, we hypothesized that beta-band modulations within lateral central regions relate to implicit sensorimotor adaptation, whereas beta-band activity within medial motor areas reflects strategic re-aiming. To test this idea, we recorded EEG activity while participants performed ballistic movements and a visuomotor rotation was introduced during short series of trials. Participants were instructed in advance about the nature of the visual perturbation and were trained to counter it by aiming at a neighboring target (i.e., re-aiming strategy). To separate beta-band activities within lateral central and medial frontal regions, we used independent component analysis (ICA). Consistent with our hypothesis, we found contrasting patterns of modulation for the two spatially distinct beta-band activities. In lateral regions, early delay beta power was decreased when a change in the visual condition required sensorimotor remapping, whereas medial frontal beta power was selectively reduced for trials in which participants were required to use the re-aiming strategy.

\section{Materials and Methods}

\section{Participants}

A total of 24 healthy adults ( 8 females; age 26.5 years; range $20-32$ years) took part in the study. All participants were right-handed, as assessed by the Edinburgh Handedness Inventory (Oldfield, 1971), and all had normal or corrected-to-normal vision. All participants were free of known neurological or psychiatric disorders and gave informed consent according to a protocol approved by the Ethics Board of the Aix-Marseille University.

\section{Experimental setup}

The experiment was performed using a robotic exoskeleton (KINARM, BKIN Technologies) that allows recording flexion and extension movements of the elbow and shoulder joints in the horizontal plane. Participants were seated with their right arm installed in the exoskeleton; the height of the chair was adjusted so that the shoulder was abducted by $\sim 70^{\circ}$. The rotation of the visual feedback of the hand was applied using a semisilvered mirror preventing direct vision of the hand. A cursor representing participants' index fingertip and the visual display were projected onto the same plane as the (invisible) hand. Participants maintained their right hand in a pointing position. Head movements were limited by using a chin rest.

\section{Task}

Participants were required to make ballistic movements with no online corrections. The starting position was indicated by a $0.75-\mathrm{cm}$-diameter white circle located at the center of a large concentric blue ring ( 10 and 14 $\mathrm{cm}$ radius for the inner and outer contour, respectively). Throughout the experiment, three possible targets located $5 \mathrm{~cm}$ away from the starting position were indicated as $0.3 \mathrm{~cm}$ diameter dark gray circles: $50^{\circ}, 80^{\circ}$ or $110^{\circ}$ from the $0^{\circ}$ straight-ahead direction (Fig. $1 A$ ).

To initiate a trial, participants had to maintain their index finger in the start circle for $2000 \mathrm{~ms}$, after which they were warned to get ready (Ready signal): the start circle disappeared and the target was indicated (one of the three targets turned from a gray to a white circle). Following a 1500 ms delay, the target was filled in white ("turned on"), indicating that the movement could be initiated (Go signal). Importantly, participants were clearly informed that they were not performing a reaction-time task and that they should take all the time they needed to prepare their movement.

Participants were instructed to move through ("shoot") the target without stopping and to end their movement between the inner and the outer contour of the concentric ring. They were also required to move fast enough so that their hand moved $5 \mathrm{~cm}$ away from the start position within $250 \mathrm{~ms}$, computed from the time when its speed exceeded $5 \mathrm{~cm} / \mathrm{s}$. Participants received visual feedback about their performance at the time the fingertip cursor reached $5 \mathrm{~cm}$ away from the starting position, hitting or missing the target: (1) the target exploded when the movement was fast and accurate enough (target hit); (2) the target turned red when the movement was fast enough but not accurate enough (target miss); and (3) the target turned green when the movement was too slow, independent of its accuracy.

To avoid online movement corrections, the fingertip cursor was turned off when the hand crossed the $10 \mathrm{~cm}$ radius inner contour of the ring. Upon movement end, the arm was passively brought back by the robot to the start position. The fingertip cursor and the starting-position circle reappeared only when the hand was back in its initial position. Each trial lasted $\sim 7 \mathrm{~s}$.

Participants were asked to keep their eyes fixed on the aimed target throughout each trial. As we explain below (see Experimental protocol), in some trials ("Rotation-Strategy" trials), the aimed target did not correspond to the target that was turned on (filled in white), but to one of its neighboring targets instead.

\section{Experimental protocol}

The experiment was made up of two sessions (preliminary and experimental) run on 2 different days, during which participants performed 
A

no-rotation trials rotation trials
without strategy (R-noS) (R-S1, R-S2, R-S3)

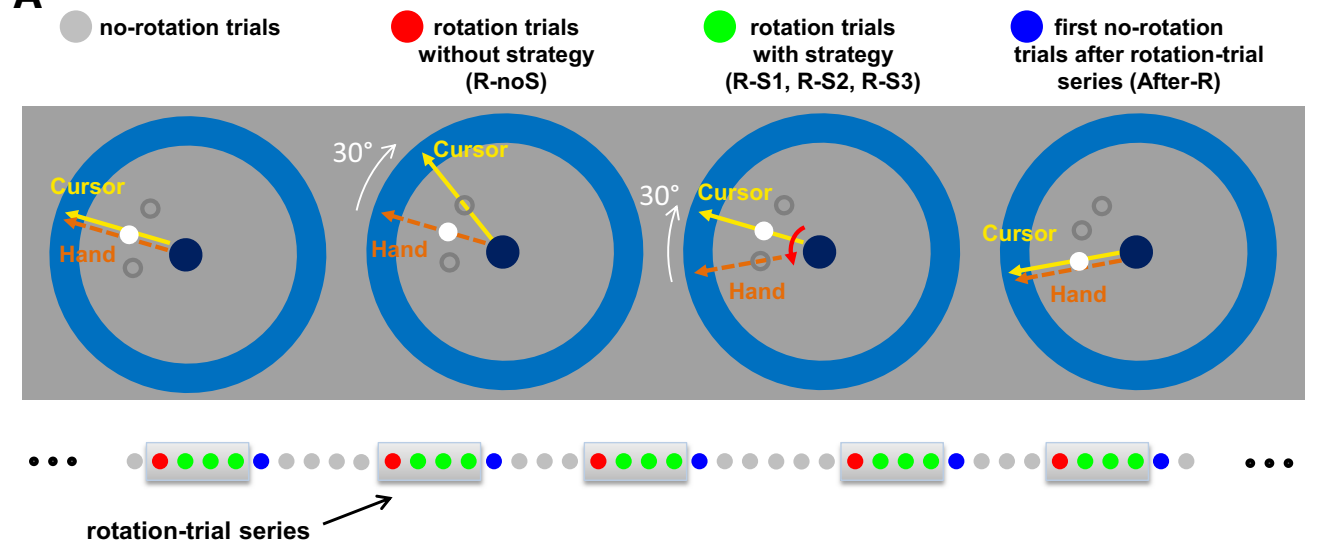

B

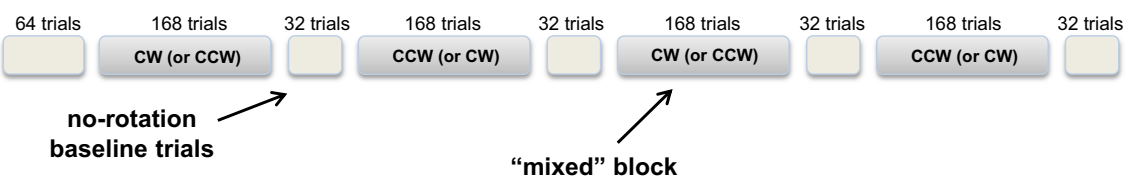

Figure 1. Task and experimental protocol. $A$, Participants were instructed to "shoot," without stopping, one of three possible visual targets. In rotation-trial series, the cursor representing the index fingertip was displayed rotated by $30^{\circ} \mathrm{CW}$ or $30^{\circ} \mathrm{CCW}$, relative to its real position. The rotation-trial series always comprised 4 trials and were separated by a variable number of no-rotation trials (gray) in which the cursor displayed the real position of the hand. In the first rotation trials (red; $R$-noS trials), the visual rotation was unexpectedly (re)introduced. In the three following trials ( $g r e e n ; R-S 1, R-S 2, R-S 3$ trials), participants knew the rotation would be applied and used the re-aiming strategy to counter it. In these trials, a red arrow was displayed as a hint for re-aiming at the appropriate neighboring target. Participants were also instructed that, after the fourth rotation trials ( $R$-S3), the visual rotation would be removed. Before the postrotation trial (After-R trials, in blue), the arrow was removed as a hint to quit the re-aiming strategy. In the After- $R$ trials, the illuminated target was always the same as the re-aimed neighboring target in the three previous strategy trials. Each "mixed" block comprised 18 rotation-trial series. $\boldsymbol{B}$, In the experimental session, participants performed four "mixed" blocks, in which the direction of the visual rotation was kept constant: $30^{\circ} \mathrm{CW}$ or $30^{\circ} \mathrm{CCW}$. The rotation directions alternated over the four "mixed" blocks, whose order was counterbalanced across participants. The "mixed" blocks were preceded by a block of 64 no-rotation trials and separated by blocks of 32 no-rotation trials.

two types of blocks: Baseline blocks comprising only unperturbed (norotation) trials and Mixed blocks in which a visual rotation $\left(+30^{\circ}\right.$ or $-30^{\circ}$ ) was applied in selected trials.

Mixed blocks structure. We designed Mixed blocks (Fig. 1A) in which short series of rotation trials alternated with no-rotation trials. The rotation-trial series always consisted of 4 movements to the same target, whereas the number and direction of no-rotation trials interleaved in between varied pseudo-randomly (at least 4 successive no-rotation trials toward one of the three targets). That is, participants could not predict when a new 4-rotation-trial series would start and thus were not expected to use the re-aiming strategy in the first trial in which the rotation was reintroduced ( $R-n o S$ trials). However, participants were informed (during the Preliminary session) about the properties of the 4-rotation-trial series; that is, they knew the visual rotation would be applied in the three following trials as well ( $R-S 1, R-S 2, R-S 3$ trials). In addition, in these trials, a red arrow (Fig. 1A) was displayed prompting them to apply the re-aiming strategy. Participants also knew that the rotation would be removed after 4 trials; that is, in the trials immediately following a 4-rotation-trial series (After- $R$ trials), they would have to quit the strategy and aim again directly at the target that was illuminated. At the end of the $R-S 3$ trials, the red arrow was removed, which ensured participants did not forget quitting the strategy. Here, it is critical to point out that the blocks of trials were designed so that the target in the After- $R$ trials always coincided with the neighboring target that was aimed at in the preceding $R-S 1, R-S 2, R-S 3$ trials, such that the explicitly (re)-aimed target remained unchanged.

In half of the no-rotation trials (in the Mixed and Baseline blocks), the target was the same as in the previous trial; for the other half, a movement-direction change was required. Each Mixed block comprised 18 rotation-trial series, and 96 no-rotation trials pseudo-randomly distributed in between, for a total of 168 trials. The direction of the rotation, clockwise $\left(30^{\circ} \mathrm{CW}\right)$ or counterclockwise $\left(30^{\circ} \mathrm{CCW}\right)$, applied in the 4-rotation-trial series was kept constant throughout each Mixed block, but reversed for each new Mixed block. Half of the participants started with a $30^{\circ} \mathrm{CW}$ Mixed block, the other half with a $30^{\circ} \mathrm{CCW}$ Mixed block.

Preliminary session (familiarization and learning of the re-aiming strat$e g y)$. During the first session, participants received verbal instructions about the general task requirements. They performed at least 4 blocks of 20 trials with no visual rotation, followed by a block in which, after 4 no rotation trials, the visual rotation (CW or CCW, counterbalanced across participants) was unexpectedly introduced for 5 trials. After participants had experienced the visual rotation, the experimenter explained in detail the nature of the perturbation and how to counter it by aiming at the (CW or CCW) neighboring target, instead of the target that was turned on. They were also presented with a red arrow (beside the startingposition circle, from the Ready signal to the end of the trial; Fig. 1A), indicating to which neighboring target they should aim to counter the rotation. Then, participants were informed about the structure of the Mixed blocks they would have to perform, in which short series of trials would alternate with no-rotation trials (detailed below). They performed two Mixed blocks, each followed by a 32-trial Baseline block (400 trials in total). EEG signals were not recorded during this session.

Experimental session. During the second session, after a 64-trial Baseline block, participants performed four Mixed blocks, each followed by a 32-trial Baseline block (864 trials in total). EEG signals were recorded throughout the session (Fig. 1B).

Between each block of trials (Mixed and Baseline) and after the 84th trial of each Mixed block, a $\sim 2$ min break was allocated. The preliminary session lasted $\sim 1 \mathrm{~h} 30 \mathrm{~min}$ in total (including robot calibration), and the experimentation session (including EEG-electrode placement and location recording) lasted $\sim 3 \mathrm{~h}$ in total.

\section{Behavioral data recording and analyses}

Angular position and velocity data of the motor resolvers were collected at $1000 \mathrm{~Hz}$. Signals were downsampled offline to $100 \mathrm{~Hz}$, and then filtered with a second-order zero-phase-shift low-pass Butterworth filter 
(cutoff frequency of $10 \mathrm{~Hz}$ ). Hand position and velocity were calculated from these angular data. Kinematic data were analyzed using custom routines written in MATLAB (The MathWorks). Trials in which the hand was not maintained stable enough in the start position during the delay between the Ready and Go signals (tangential velocity $>6 \mathrm{~cm} / \mathrm{s}$ ), or in which the movement was initiated before the Go signal, were excluded from the analyses $(\sim 1 \%$ of trials). Movement onset was defined as the time when the tangential velocity exceeded $5 \mathrm{~cm} / \mathrm{s}$. The movement offset corresponded to the time when tangential velocity fell below $5 \mathrm{~cm} / \mathrm{s}$ and remained below this value for at least $1500 \mathrm{~ms}$. To quantify kinematic errors, we computed the perpendicular deviation, from the straight line that connects the starting position to the target, at maximum velocity (PD-vel). This measure quantifies error in initial movementdirection (feedforward component) and captures adaptive update of the motor-command.

In addition, movement duration and the delay between the Go signal and movement onset were calculated for the different types of trials. Trials that were performed too slowly $(\sim 4 \%)$ were excluded from the analyses.

For the statistical analyses, we considered $R-n o S, R-S 1, R-S 2, R-S 3$ and After-R trials of the Mixed blocks, and the no-rotation trials of the Baseline blocks, which we used as reference. To correct for individual movement-trajectory biases, for each participant, we subtracted from the PD-vel observed in $R$-noS, $R$-S1, R-S2, R-S3 and After-R trials the mean of the $\mathrm{PD}$-vel computed over the 32 no-rotation trials of the Baseline block following them. To collapse data from different Mixed blocks, with opposite visual rotations $\left(30^{\circ} \mathrm{CW}\right.$ vs $\left.30^{\circ} \mathrm{CCW}\right)$, we set the signs of the $\mathrm{PD}$-vel values so that hand-path deviations in the direction of the visual rotation corresponded to positive values. Previously, we conducted preliminary analyses to test for differences between the movement errors induced by the two rotation directions.

Repeated-measures univariate ANOVAs, followed by post hoc pairwise $t$ tests, were run on kinematic-error measures (PD-vel), movement durations, and Go-movement-onset delays. In all cases, trial category $(R-$ noS, $R-S 1, R-S 2, R-S 3$ and After-R) was used as within-subject factor. Normality and sphericity assumptions were controlled and Huynh-Feldt correction applied whenever appropriate. For all tests, the significance threshold was set to 0.05 , and multiple comparisons corrections were performed with the Bonferroni procedure. For post hoc tests, $p$ values presented in Results are multiplied accordingly.

\section{EEG data recording and analysis}

EEG activity was recorded continuously at $1024 \mathrm{~Hz}$ using a 64-channel ActiveTwo system (BioSemi) referenced to the Common Mode Sense/ Drive Right Leg (CMS/DRL) contact. Electrodes were embedded into an elastic cap and distributed over the scalp according to the extended 10-20 EEG system. The electrode offsets, the voltage differences between the CMS and each active electrode, were monitored to remain within $\pm 20 \mu \mathrm{V}$. For each participant, electrode locations and nasion and preauricular points were recorded by an infrared camera (Rogue Research). EOG activity was recorded with surface electrodes placed near both outer canthi (saccades) as well as under and above the right orbit (blinks).

EEG preprocessing. EEG data were preprocessed using the free software ELAN (Aguera et al., 2011), which allows especially good visual inspection of the raw EEG signals for artifact rejection purposes. Continuous signals were rereferenced to the average of all electrodes, filtered between 2 and $70 \mathrm{~Hz}$ (Butterworth order 2) and downsampled to $256 \mathrm{~Hz}$. Nonstereotypical artifacts (that cannot be captured by ICA) (see Makeig et al., 1997; Delorme et al., 2007) were identified and rejected upon visual data screening. Further analyzes were run using the free and open-source software FieldTrip (Oostenveld et al., 2011).

ICA. The preprocessed EEG signals were cut into time segments extending from -4000 to $3000 \mathrm{~ms}$ with respect to movement onset, which covered approximately the complete trials, slightly variable in duration. The epoched EEG data were then submitted to ICA (runica algorithm).

EEG time-frequency analysis. Time-frequency analyses were performed on the time courses of the independent components (ICs). Single-trial signals were transformed in the time-frequency domain by convolution with the complex Morlet's wavelets characterized by the ratio $\mathrm{f} 0 / \sigma \mathrm{f}=7$, with $\mathrm{f} 0$ ranging from 2 to $50 \mathrm{~Hz}$ by steps of $0.5 \mathrm{~Hz}$. To calculate the event-related changes in beta power, the raw power data were log-transformed and then normalized relative to the average power calculated over all trials, as no clear baseline period could be defined during our task (Tan et al., 2014; Torrecillos et al., 2015; Alayrangues et al., 2019). For each participant and each time point (50 ms bin), power was averaged over trials within a specific beta frequency band (individually selected; see below) and smoothed using a Gaussian kernel with 7 time points ( $350 \mathrm{~ms})$ FWHM.

IC selection. Our goal was to identify for each participant two different ICs that would capture distinct beta-band activities: medial frontal betaband activity, which we hypothesized would reflect processes recruited for the explicit re-aiming strategy, on the one hand; and beta-band activity from lateral central regions, modulated during the foreperiod in relation to implicit sensorimotor adaptation processes (Torrecillos et al., 2015, Alayrangues et al., 2019), on the other hand.

For the IC selection, we proceeded in two steps (Alayrangues et al., 2019). First, we preselected ICs based on their topographies. For this step, we defined spatial ROIs; ICs that exhibited the largest weighting within one of these ROIs were preselected. To capture medial frontal beta-band activity, we considered an ROI, including electrodes F1-Fz-F2-FC1-FCzFC2-C1-Cz-C2; and to capture beta-band activity lateralized over the left hemisphere, we used an ROI, including electrodes C3-C5-CP1-CP3CP5-P1-P3-P5. For 1 participant (s18; see Fig. 3A), we made an exception selecting an IC with maximum weighting at electrode FC3. Then, in a second step, we examined the time-frequency representation of the time courses of the preselected ICs to retain for each participant one IC of each type. For this step, within the trial period going from -2.5 to $1.5 \mathrm{~s}$ relative to movement onset, we examined the time-frequency representations of the time courses of the preselected ICs computed over all trials. For each individual and type of ICs, we selected the IC (most of the time, only one IC per participant was preselected) and the frequency-band, between 17 and $40 \mathrm{~Hz}$, exhibiting the largest beta-power variance. Betapower profiles were then obtained by averaging $>5-\mathrm{Hz}$-wide frequency bands centered about the individually selected frequency. We defined the frequency bands of interest on an individual basis, an approach used to take into account the substantial interindividual differences in the betafrequency bands exhibiting the most prominent movement-related power changes (e.g., Little et al., 2013; Torrecillos et al., 2015; Tinkhauser et al., 2017; Alayrangues et al., 2019; Meidahl et al., 2019).

We estimated the percentage of variance in the group average (all trials of all participants) EEG-sensor beta-power profiles computed over the medial and the lateral ROIs accounted for by the selected medial and the lateral central ICs, respectively. In both cases, we computed the variance explained $\left(R^{2}\right)$ for two time windows: (1) including the premovement and postmovement periods, -3000 to $2500 \mathrm{~ms}$ relative to movement onset; and (2) restricted to the premovement period, -3000 to $0 \mathrm{~ms}$ relative to movement onset. The medial frontal ICs accounted for $55 \%$ and $87 \%$ of the variance in the medial-ROI group average beta-power profiles, for the two time windows, respectively. The lateral central ICs accounted for $24 \%$ and $88 \%$ of the variance in the medial-ROI group average beta-power profiles, for the two time windows, respectively.

Statistical analyses of the beta-power profiles of the ICs. We analyzed the signals aligned to movement onset within the time window extending from -1.5 to $0 \mathrm{~s}$ relative to movement onset. Using custom-written MATLAB (The MathWorks) routines, statistical analyses were computed, at each time point ( $50 \mathrm{~ms}$ bin), on the beta power of the time courses of the ICs. Significance level was set to 0.05 . The false discovery rate (FDR) method was used to correct for multiple comparisons along the time axis, and the Bonferroni procedure was used to correct for multiple pairwise post hoc comparisons, following significant repeatedmeasures ANOVAs.

For all statistical tests, repeated-measures ANOVAs, and paired $t$ tests, contrasting the different categories of trials ( $R-n o S, R-S 1, R-S 2, R-S 3$ and After- $R$ of the Mixed blocks, and the trials of the Baseline blocks), the number of trials was equated across the different categories and included in the analyses only the data of participants for which at least 20 trials, per trial category, remained after artifacts rejection. 
Dipole fitting of the ICs. To further test the hypothesis that the two distinct beta-band activities were generated by distinct neural substrates, we conducted dipole source analysis of the lateral central and medial frontal ICs. For each participant, an equivalent current dipole model was computed for the two selected ICs by using the ' $\mathrm{ft}$ _dipolefitting' FieldTrip function. Informing the forward model of the recorded locations of the EEG electrodes, dipoles were localized within a three-shell boundary element model (BEM) of the MNI standard brain. For all identified individual ICs, only dipole solutions with residual variance $<10 \%$ were considered.

\section{Results}

Behavioral performance

One participant was excluded from all analyses (behavioral and EEG) because of noisy EEG data; hence, data from 23 participants were analyzed. As expected, for the different categories of trials, $R$-noS, $R-S 1, R-S 2, R-S 3$, and After-R, kinematic errors of different sizes and directions were observed. This is illustrated in Figure $2 A$, showing hand-path perpendicular deviations measured at maximum tangential velocity (PD-vel) for all trials averaged across participants. Statistical analyses were performed on the PD-vel values corrected for individual movement-trajectory biases and collapsed across Mixed blocks (see Material and Methods). Data were collapsed following preliminary analyses revealing no significant difference between the effects observed for the opposite $30^{\circ} \mathrm{CW}$ and $30^{\circ} \mathrm{CCW}$ rotations. Indeed, a 2 (rotation direction) $\times 5$ (type of trials) two-way repeatedmeasures ANOVA revealed no main effect of rotation direction $\left(F_{(1,22)}=2.260, p=0.134\right)$ and no significant interaction effect $\left(F_{(4,88)}=0.743, p=0.564\right)$, although a significant main effect of type of trials was observed $\left(F_{(4,88)}=247.1, p<0.0001\right)$.

Group data are presented in Figure $2 B$. Large kinematic errors in the direction of the visual rotation were observed for $R$-noS trials, confirming that participants did not predict when the perturbation would be reintroduced. Clear initial movementdirection errors were also visible for the After-R trials, with deviations in the opposite direction attributable to implicit sensorimotor adaptation aftereffects.

A repeated-measures ANOVA on the PD-vel confirmed a significant effect of the category of trials $\left(F_{(4,88)}=240.7, p<\right.$ $0.0001)$. Post hoc comparisons revealed that the hand-path deviations in $R$-noS trials and After- $R$ trials differed from all other types of trials. For $R-n o S$ versus $R-S 1, R-S 2, R-S 3$, and After-R, respectively: $t_{(22)}=16.721, p<0.0001 ; t_{(22)}=16.2511, p<$ $0.0001 ; t_{(22)}=14.9879, p<0.0001 ; t_{(22)}=37.5523, p<0.0001$. For After- $R$ versus $R-S 1, R-S 2$ and $R-S 3$, respectively: $t_{(22)}=$ $-6.2435, p<0.0001 ; t_{(22)}=-9.1947, p<0.0001 ; t_{(22)}=$ $-9.3784, p<0.0001$; with deviations in the direction of the visual rotation for $R$-noS trials, and deviations in the opposite direction for After- $R$ trials. Kinematic errors did not differ significantly between the rotation-strategy trials, $R-S 1, R-S 2, R-S 3$. For $R-S 1$ versus $R-S 2: t_{(22)}=-1.8821, p=0.7310$; for $R-S 1$ vs $R-S 3: t_{(22)}=$ $-2.4834, p=0.2110$; for R-S2 vs R-S3: $t_{(22)}=-2.3240, p=$ 0.2980 . In addition, for the rotation-strategy trials, we conducted preplanned comparisons (uncorrected $p$ values) to test whether kinematic errors differ significantly from zero (i.e., from Baseline trials). No significant difference was found $\left(t_{(22)}=0.08, p=\right.$ $0.937 ; t_{(22)}=1.26, p=0.221 ; t_{(22)}=1.76, p=0.092$, for $R-S 1$, $R-S 2$, and $R-S 3$ trials, respectively), confirming that, in general, participants properly applied the re-aiming strategy. However, reaches in the latter trials were substantially more variable than in the Baseline trials; while participants properly hit the target (success) in $49.96 \%$ of the Baseline trials, they did so in $17.59 \%$, $18.52 \%$, and $18.63 \%$ of $R-S 1, R-S 2, R-S 3$ trials, respectively (in
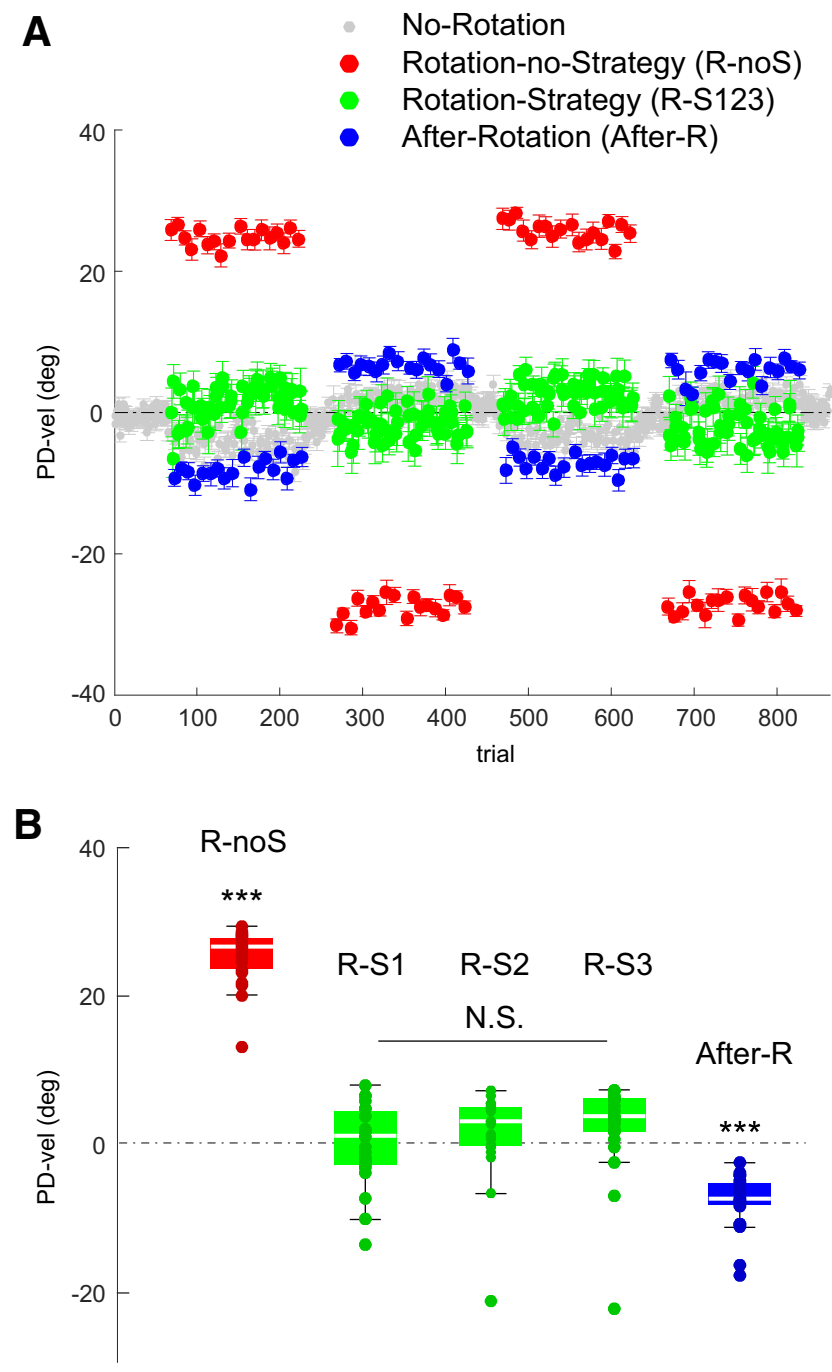

Figure 2. Behavioral data. $\boldsymbol{A}$, Initial movement-direction error data averaged across partic ipants $(n=23)$, calculated as the perpendicular hand-path deviation measured at tangential velocity peak (PD-vel). Error bars indicate SE. Colors used for the individual trials represent the different trial categories (see Fig. 1). Positive values correspond to errors in the CW direction. $\boldsymbol{B}$, The values of the perpendicular deviation at velocity peak (PD-vel) observed at the group level $(n=23)$ for the different trial categories, $R-n O S, R-S 1, R-S 2, R-S 3$, and After- $R$. These values have been corrected for individual biases measured in the Baseline trials. The sign of the errors observed for the opposite visual rotation (CW and CCW) has been flipped so that data could be collapsed (see Materials and Methods). Box plot represents statistical results. Middle mark indicates the median. Edges of the box represent the 25th and 75th percentiles. Whiskers extend to the extreme data. Each participant's data are plotted individually. Kinematic errors observed for the $R$-noS and After- $R$ trials significantly differed from those observed in all other trial categories. ${ }^{* *} p<0.0001$ (Bonferroni-corrected). No significant difference was observed between the strategy trials, $R-S 1, R-S 2$, and $R-S 3$.

R-noS trials and After-R trials, respectively, $0 \%$ and $16.61 \%$ of successes were observed).

In addition, we analyzed movement durations and delays between the Go signal and movement onset (movements were initiated without time pressure). For movement durations, repeated-measured ANOVAs (within 6 categories of trials, including the Baseline trials) revealed a significant effect of trial category $\left(F_{(5,110)}=4.33, p=0.0012\right)$. Post hoc pairwise comparisons indicated that R-NoS trials had significantly longer durations than Baseline trials $\left(t_{(22)}=3.9507, p=0.0095\right.$; Baseline: $572 \pm 83 \mathrm{~ms}, R-N o S: 606 \pm 101 \mathrm{~ms}$ ). No other pairwise contrast survived multiple comparison correction $(R-S 1: 584 \pm 96 \mathrm{~ms}$, 


\section{A Topographies}
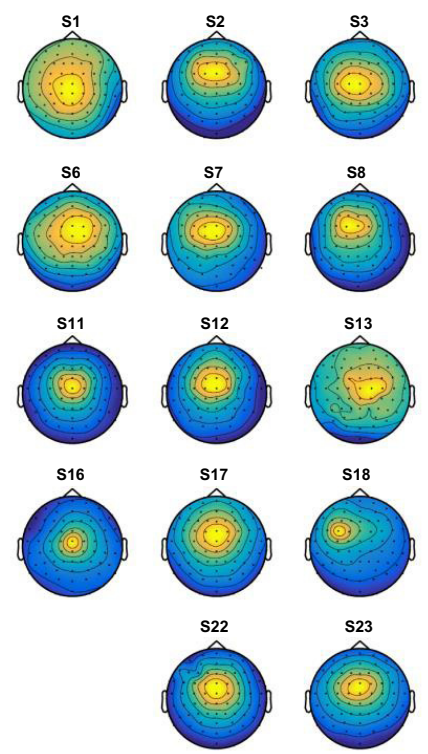
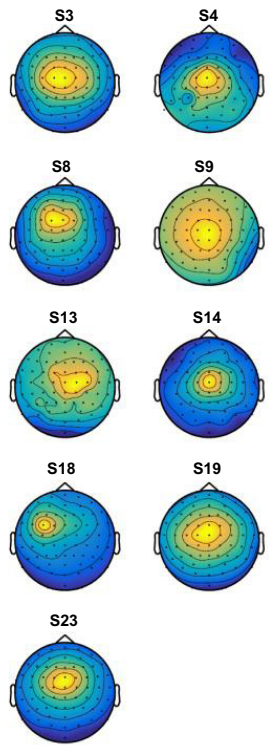

\section{B Time-frequency representations}

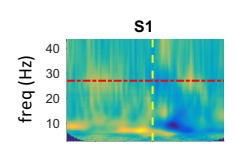

s6
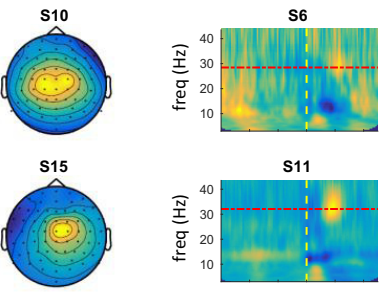

s11
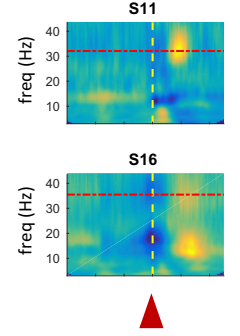

movement onset

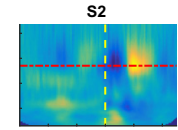

s7

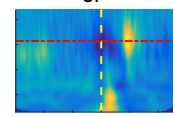

S12

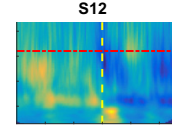

$\$ 17$

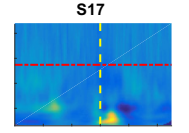

S22

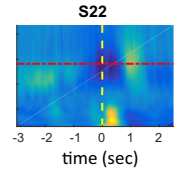

s3

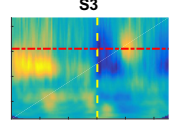

s8

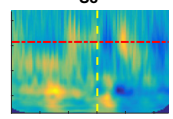

s13

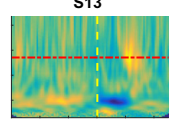

S18

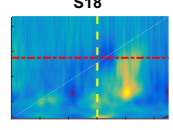

S23

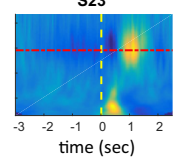

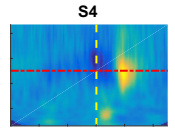

S9

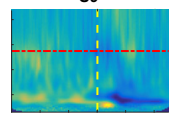

S14

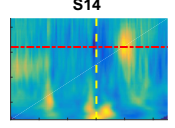

S19

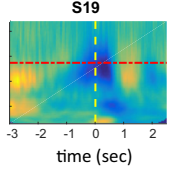

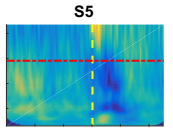

S10
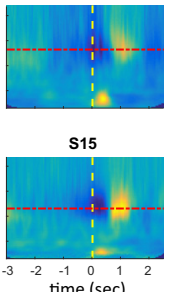

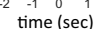

Figure 3. Individual medial frontal ICS. A, Topographies of the medial frontal ICs identified for each participant. $\boldsymbol{B}$, Time-frequency representations of the time courses of the individual ICs aligned to movement onset. Red dotted lines indicate the frequency bands selected for each participant.

R-S2: $579 \pm 85 \mathrm{~ms}, R-S 3: 577 \pm 88 \mathrm{~ms}$, After-R: $586 \pm 99 \mathrm{~ms}$ ), whereas delays between the Go signal and movement onset did not differ significantly between trial categories $\left(F_{(5,110)}=0.16\right.$, $p=0.977$; Baseline: $641 \pm 204 \mathrm{~ms}, R-N o S: 638 \pm 234 \mathrm{~ms}, R-S 1$ : $650 \pm 222 \mathrm{~ms}, R-S 2: 639 \pm 217 \mathrm{~ms}, R-S 3: 647 \pm 225 \mathrm{~ms}$, After-R: $643 \pm 251 \mathrm{~ms})$.

In summary, first, the behavioral data confirmed that participants were able to properly use the re-aiming strategy. When participants knew they would have to counter a visual rotation ( $R-S 1-3$ trials), cursor trajectories did not significantly differ from those in the Baseline trials, even though increased directional variability was observed. Second, substantial aftereffects were observed upon removal of the visual rotation (After- $R$ trials), which confirmed that implicit sensorimotor remapping did occur over the short rotation-trial series.

\section{EEG time-frequency data}

Figures 3 and 4, respectively, present the topographies and timefrequency maps of the medial and lateral central ICs selected for each participant. As indicated in these figures, medial and lateral central ICs could be identified in 21 of 23 and 20 of 23 participants, respectively (empty spaces indicate the participants for whom the corresponding IC could not be identified). One may notice that, as visible in Figure 3, for a good proportion of the participants, the lateral central ICs exhibited topographies with a steepest gradient overlying the central area, and aligned to the central sulcus, suggesting a tangential source in the anterior or posterior bank of the central sulcus.

The frequency bands selected for each participant are shown (red lines) on the corresponding time-frequency maps (Figs. 3B, $4 B$ ). Individual frequency bands selected for the two types of ICs differed significantly, with higher frequency bands selected for the medial than for the lateral central ICs $\left(t_{(18)}=7.1621, p<\right.$ 0.001 ; mean \pm SE: $28.19 \pm 0.75 \mathrm{~Hz}$, and $21.33 \pm 0.44 \mathrm{~Hz}$, for the medial and lateral central ICs, respectively).
As can be seen in Figures 5 and 6, for both types of ICs, beta power was clearly modulated in relation to the reaching movements. For the Baseline trials, a transient beta-power enhancement could be observed $\sim 1 \mathrm{~s}$ before movement onset. For all trials, beta power dropped during movement and exhibited a clear rebound at movement end.

In both types of ICs, we analyzed the signals within the time window extending from -1.5 to $0 \mathrm{~s}$ relative to movement onset (highlighted in gray in Figs. 5, 6).

\section{Beta-power profiles of the medial frontal ICs}

Our first goal was to assess whether medial frontal beta-band activity during movement preparation reflected the activation of the explicit re-aiming strategy. To this aim, we compared the beta-power profiles observed for the strategy trials with those obtained for trials in which participants knew they would not have to counter a visual rotation.

In a first step, at each sampling point of the time window of interest, we conducted a repeated-measures ANOVA on the betapower levels computed for the rotation-strategy $(R-S 1, R-S 2$, and $R$-S3; pooled, given the absence of significant kinematic difference between them), After-R, and Baseline trials, respectively. For these tests, we controlled for the proportion of failed and successful trials across the different categories of trials, which differed substantially as for the outcome of the task (rate of successful trials: $18.25 \%, 16.61 \%$, and $49.96 \%$ ), for strategy (R-S1, R-S2, and $R-S 3$, pooled), After-R, and Baseline trials, respectively. We did so since preliminary analyses (results not shown) revealed that beta power during movement preparation slightly differed depending on the success (accuracy) of the forthcoming movement. Specifically, when contrasting successful and failed Baseline trials, we observed a tendency (not statistically significant though) for successful trials to exhibit lower beta power. We conducted two complementary analyses in which the rate of failures and successes was equated across the different categories of 


\section{A Topographies}
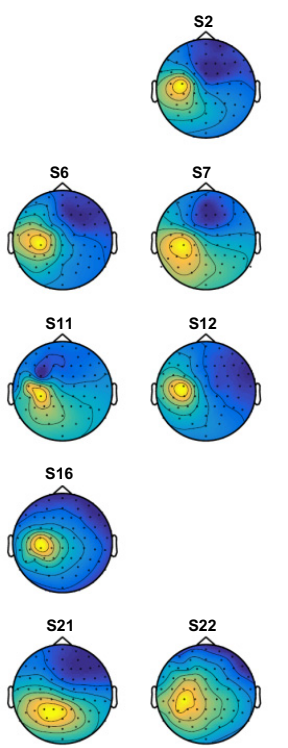
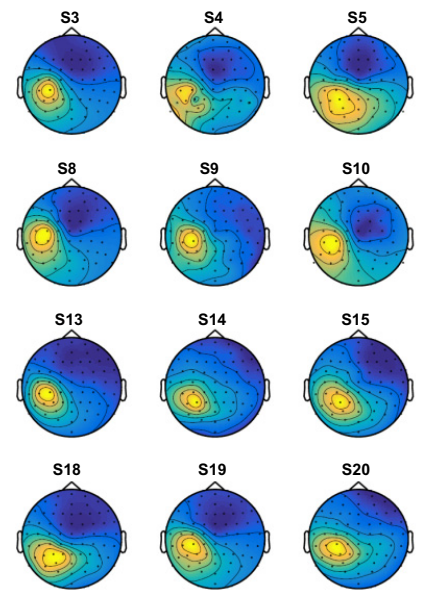

\section{B Time-frequency representations}

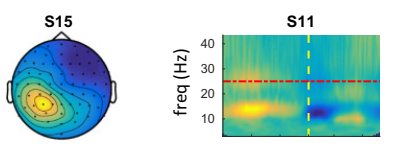

s16

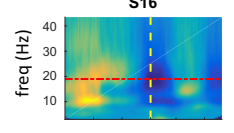

S21

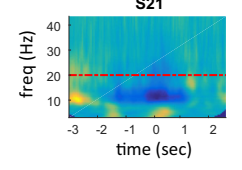

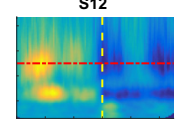

$\Delta$ movement onset

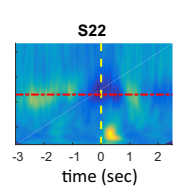

s3

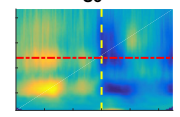

s8

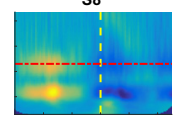

S13

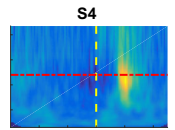

s9
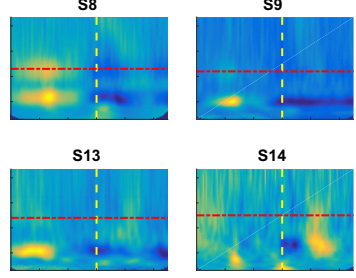

S18

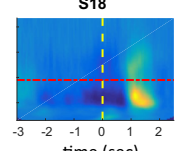

time (sec)
S14

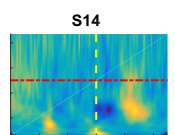

s19

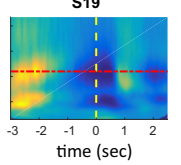

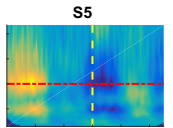

s10

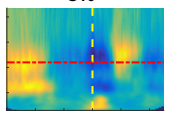

S15

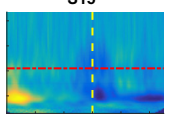

s20

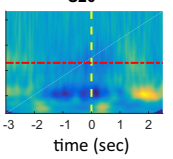

Figure 4. Individual lateral central ICS. A, Topographies of the lateral central ICs identified for each participant. B, Time-frequency representations of the time courses of the individual ICs aligned to movement onset. Red dotted lines indicate the frequency bands selected for each participant.

trials. The first analysis was conducted including failed trials only. Divergence between the beta-power profiles observed for the different categories of trials was visible (Fig. 5A). Repeatedmeasures ANOVAs, with the category of trials (rotation-strategy, After- $R$, and Baseline) as within-subjects factor (mean \pm SE number of trials for each category: $47 \pm 2.90$ ), confirmed significant effects over the trial period extending from -850 to $-300 \mathrm{~ms}$ before movement onset (FDR-corrected for multiple comparisons along the time axis). Post hoc pairwise comparisons, performed at each significant time point, revealed that beta power was significantly lower for strategy trials $(R-S 1, R-S 2$, and $R-S 3$, pooled) than for After-R and Baseline trials, respectively, from -600 to $-450 \mathrm{~ms}$ and from -850 to $-500 \mathrm{~ms}$ before movement onset (Bonferroni-corrected for multiple pairwise trial-category contrasts). No significant difference was found between the betapower profiles observed for the After-R and Baseline trials, in which participants did not use the re-aiming strategy. Similar results were obtained, for the second analysis, including failed and successful trials in the same proportion for all conditions (mean \pm SE number of trials for each category: $47 \pm 2.90$ and $11 \pm 1.18$ for failed and successful trials, respectively). Repeatedmeasures ANOVAs were significant from -850 to $-250 \mathrm{~ms}$ before movement onset (FDR-corrected for multiple comparisons along the time axis). Post hoc tests indicated significantly lower beta-power for strategy trials ( $R-S 1, R-S 2$, and $R-S 3$, pooled) than for After-R and Baseline trials, respectively, from -700 to -550 $\mathrm{ms}$ and from -850 to $-500 \mathrm{~ms}$ before movement onset (Bonferroni-corrected for multiple pairwise trial-category contrasts). Beta-power profiles for the After-R and Baseline trials did not differ significantly from each other.

In a second step, we refined the previous results by examining separately the beta-power levels for the different strategy trials, $R-S 1, R-S 2$, and $R-S 3$. Based on the previous results, we conducted preplanned one-tailed pairwise comparisons, contrasting each type of strategy trials ( $R-S 1, R-S 2$, and $R-S 3)$ and the Aftereffect trials with the Baseline trials (Fig. $5 B$ shows the results FDR- corrected for multiple comparisons along the time axis, with no Bonferroni correction for multiple pairwise contrasts.) For all three strategy trials, beta power was significantly lower than for the Baseline trials. Beta-power attenuation was, however, more pronounced for the first rotation-strategy trial than for the two following ones. For $R$-S1 trials, beta power was significantly reduced over a trial period extending from -1000 to $-150 \mathrm{~ms}$ before movement onset; whereas for $R-S 2$ and $R$-S3 trials, significant attenuation was observed over shorter time windows: from -850 to $-700 \mathrm{~ms}$ and from -400 to $-300 \mathrm{~ms}$, for $R-S 2$ trials, and from -850 to $-750 \mathrm{~ms}$ and from -250 to $-200 \mathrm{~ms}$, for $R-S 3$ trials. No difference was found between the After-R and the Baseline trials, confirming (with more powerful tests) the result of the post hoc tests presented in Figure 5A. Given the difference in the number of trials included to compute the individual beta-power curves, the beta-power profiles for After-R and the Baseline trials slightly differ between Figure $5 A$ (mean \pm SE number of trials: $42 \pm 2.39$ ) and Figure $5 B$ (mean \pm SE number of trials: $47 \pm$ 2.90).

Additionally, we looked into the possibility that the more sustained beta-power attenuation observed for the $R-S 1$ trials compared with $R-S 2$ and $R-S 3$ trials related to the fact that in $R-S 1$ trials a change in the motor output was required relative to the previous trials (execution of a movement in a different direction), which was not the case for $R-S 2$ and $R-S 3$ trials. To this aim, we contrasted the Baseline trials in which participants had to switch movement direction, relative to the previous trial, with the Baseline trials for which the target remained unchanged (mean $\pm \mathrm{SE}$ number of trials per types of trials: $81 \pm 2.01$ ). One-tailed contrasts revealed no significant difference between the two types of Baseline trials. These results suggest that the more sustained betapower attenuation observed for the $R$-S1 trials compared with $R-S 2$ and $R-S 3$ trials cannot be attributed to the change in movement direction, relative to the previous trial, required in $R-S 1$ trials and not in $R-S 2$ and $R-S 3$ trials. Rather, they suggest that the 
A

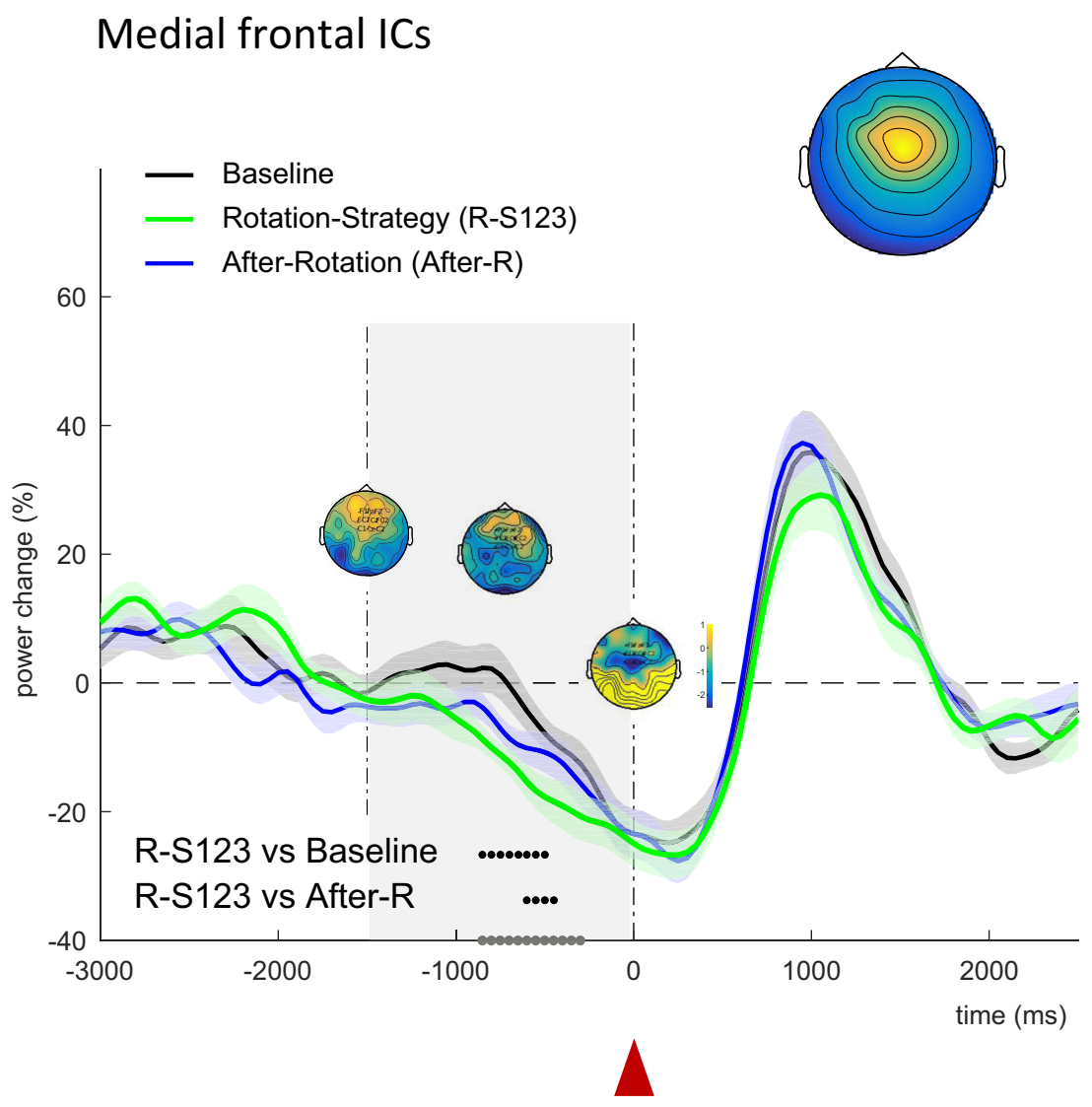

movement onset
B
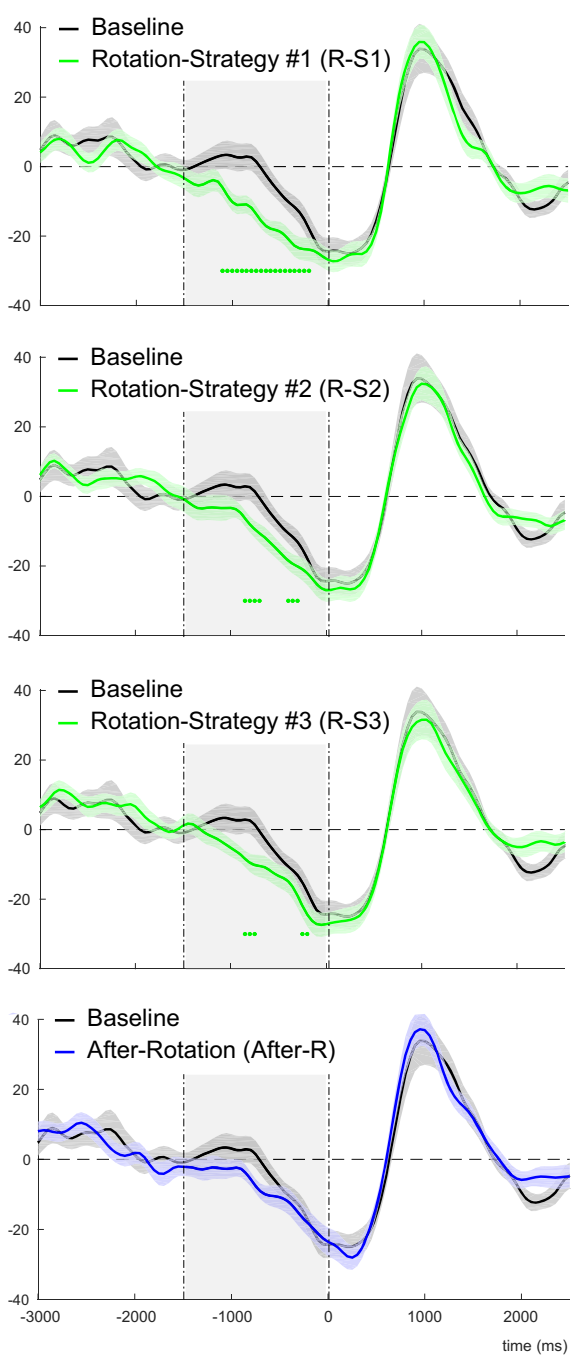

Figure 5. Beta-power modulations for the medial frontal ICs. Group average beta-power profiles for the medial frontal ICs aligned to movement onset. These were obtained by averaging over the individually selected ICs and frequency bands separately for the different categories of trials. Gray shading represents the time window of interest. $A$, Group average beta-power profiles for trial categories Baseline (black), R-S1, R-S2, and R-S3 pooled (green), and After-R (blue). Gray dots indicate the period during which beta power differed significantly (FDR-and Bonferroni-corrected; see Materials and Methods) between trial categories. Black dots indicate the periods during which pairwise comparisons were significant. Beta power for the rotation-strategy trials (R-S1, $R-S 2$, and $R$-S3, pooled) was significantly decreased relative to the Baseline and postrotation trials (After-R), which did not differ significantly from each other. Top right, The group average (time invariant) topography of the medial frontal ICS. Bottom, Group topographies for the EEG signal beta-power (frequency bands selected for the medial frontal ICs) for all trials averaged over $500 \mathrm{~ms}$ time windows centered at $-1500,-750$, and $0 \mathrm{~ms}$, respectively, relative to movement onset. Electrode FCz indicates the center of the region of interest used for selecting the individual ICs. $\boldsymbol{B}$, Top to bottom, Pairwise comparisons of group average beta power for the Baseline trials (black) and, respectively, for the rotation-strategy trials $R-S 1, R-S 2, R-S 3$, and postrotation trials (After-R). In each panel, the periods during which pairwise contrasts were significant (one-tailed FDR-corrected) are indicated by colored bars. While beta power in $R$-S1, $R$-S2, and $R-S$ was significantly decreased relative to Baseline trials, beta power for the postrotation trials (After-R) did not differ significantly from the Baseline trials. The beta-power decrease was more marked for the first rotation-strategy trials $R$ - $S 1$ than for the two following ones, $R-S 2$ and $R-S 3$.

greater beta-power attenuation in $R$-S1 trials reflects the first implementation of the re-aiming strategy.

\section{Beta-power profiles of the lateral central ICs}

In previous studies, we found that beta-band activity generated in lateral regions was modulated during the foreperiod in relation to implicit sensorimotor adaptation processes (Torrecillos et al., 2015; Alayrangues et al., 2019). Here, our aim was to investigate the pattern of modulation of this beta-band activity in a task in which both implicit and explicit error-reduction mechanisms are at work.

We contrasted the beta-power profiles for rotation-strategy ( $R-S 1, R-S 2$, and $R-S 3$ pooled), After-R trials and Baseline trials, respectively. As for the analyses conducted on the medial frontal ICs (see above), we considered failed trials only. Here, however, we report results obtained by restricting further the set of the included trials. As indicated above (see Materials and Methods), to better isolate the effect of implicit sensorimotor adaptation processes, we designed the trial sequences so that, in the After- $R$ trials, the target that participants had to explode always coincided with the neighboring target they were aiming at in the previous strategy trials; that is, no movement direction changes was required relative to the previous trials. Thus, here we used as reference Baseline trials in which the aimed target remained unchanged relative to the previous trial (a similar pattern of results was obtained by using Baseline trials with or without movement direction change; we present here the results using the more conservative comparisons, including fewer trials). As presented in Figure $6 A$, repeated-measures ANOVAs revealed significant effects of the category of trials (rotation-strategy, After- $R$ trials, 
A

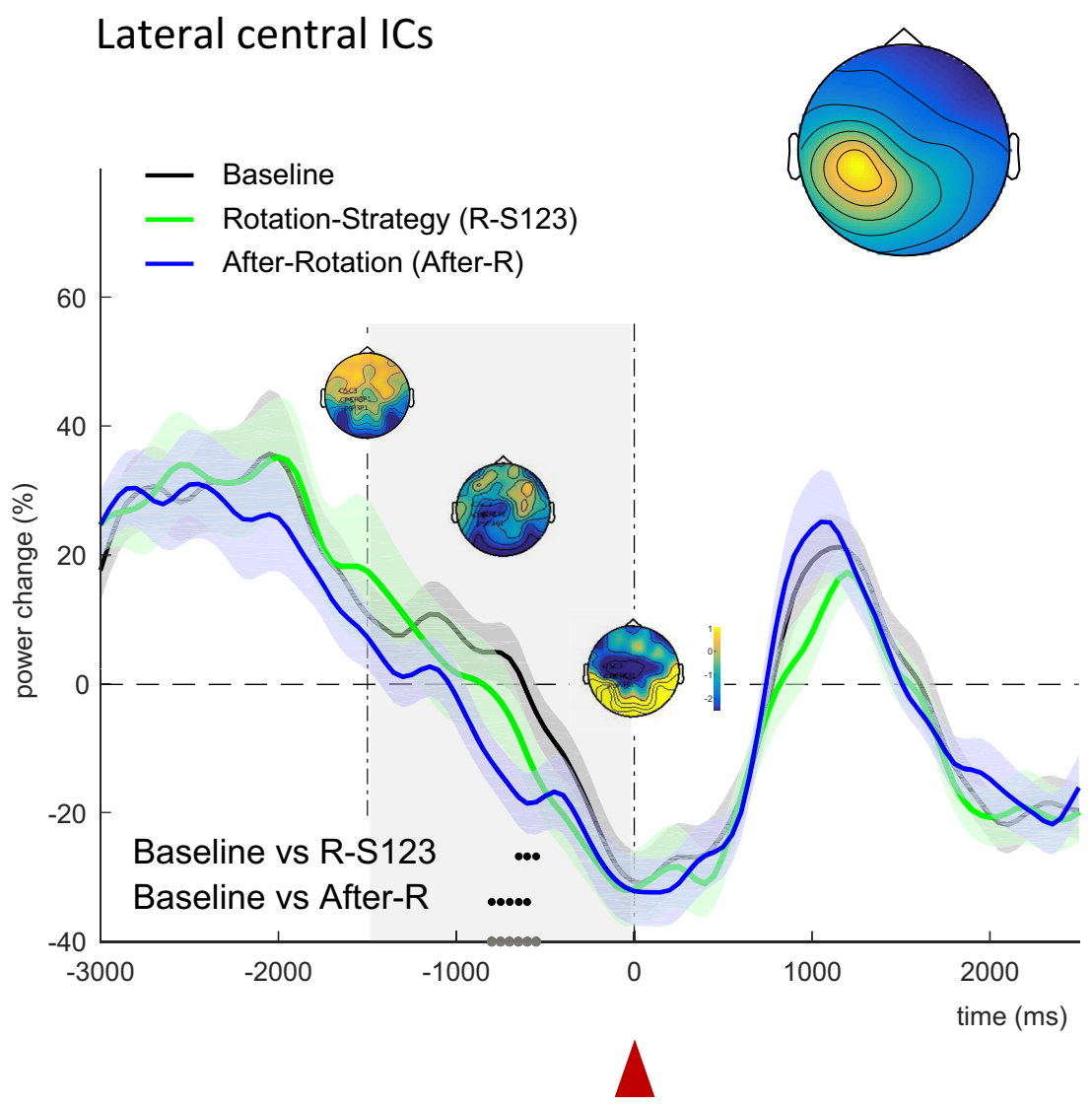

movement onset
B
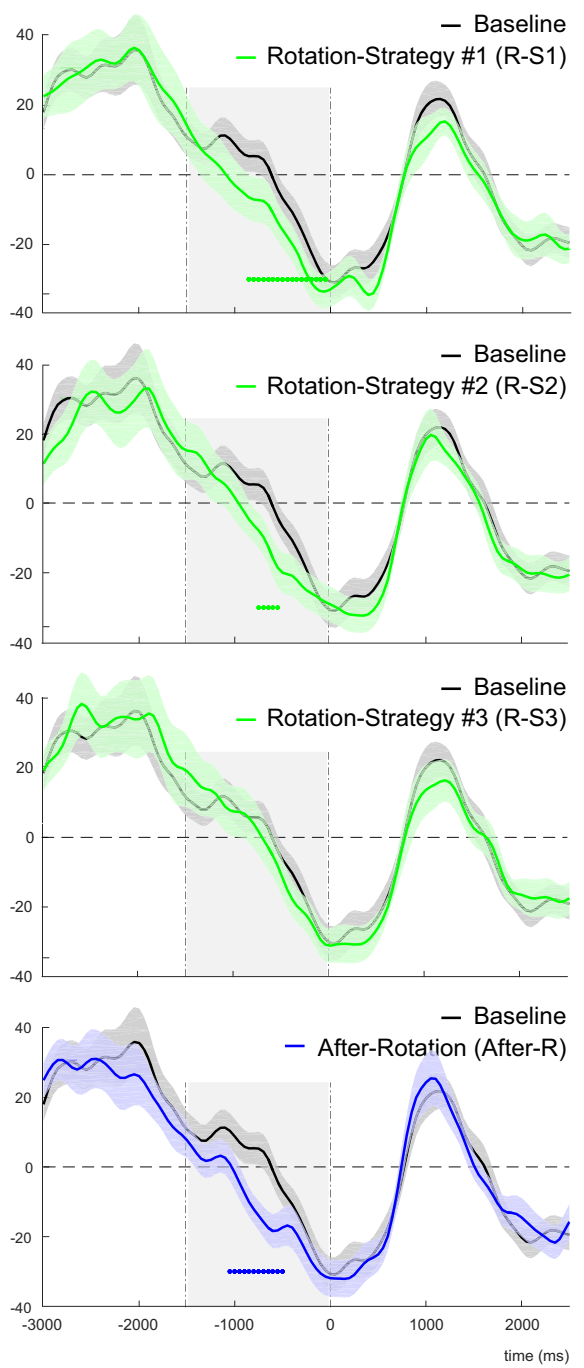

Figure 6. Beta-power modulations for the lateral central ICs. Group average beta-power profiles for the lateral central ICs aligned to movement onset. These were obtained by averaging over the individually selected ICs and frequency bands separately for the different categories of trials. Gray shading represents the time window of interest. $A$, Group average beta-power profiles for trial categories Baseline (black), $R-S 1, R-S 2$, and $R-S 3$, pooled (green) and After-R (blue). Gray dots indicate the period during which beta power differed significantly (FDR- and Bonferroni-corrected; see Materials and Methods) between trial categories. Black dots indicate the periods during which pairwise comparisons were significant. Beta power for the rotation-strategy trials (R-S1, $R-S 2$, and $R-S 3$, pooled) and for the postrotation trials (After- $R$ ) was significantly decreased relative to the Baseline trials. Top right, The group average (time invariant) topography of the lateral central ICS. Bottom, Group topographies for the EEG signal beta power (frequency bands selected for the lateral central ICs) for all trials averaged over $500 \mathrm{~ms}$ time windows centered at $-1500,-750$, and 0 $\mathrm{ms}$, respectively, relative to movement onset. Electrode CP3 indicates the center of the region of interest used for selecting the individual ICs. $\boldsymbol{B}$, Top to bottom, Pairwise comparisons of group average beta power for the Baseline trials (black) and, respectively, for the rotation-strategy trials $R-S 1, R-S 2, R-S 3$ and postrotation trials (After-R). In each panel, colored bars represent the periods during which pairwise contrasts were significant (one-tailed FDR-corrected). Significant beta-power attenuations were observed for rotation-strategy trials $R-S 1$ and $R-S 2$, as well as for the After-R trials. The attenuation was sustained for the After-R trials and the first rotation-strategy trials, $R-S 1$. A gradual decrease of the effect was observed over the strategy trials $R-S 1$ to $R-S 3$.

Baseline) on the beta-power levels over a trial period extending from -800 to $-550 \mathrm{~ms}$ (mean \pm SE number of trials per category: $38 \pm 2.59)$. Post hoc pairwise comparisons indicated that beta power was significantly lower for the strategy trials $(R-S 1$, $R$-S2, and $R$-S3 pooled) and the After- $R$ trials than for the Baseline trials. The effect, however, was more pronounced for the After- $R$ trials than for the strategy trials. Significant beta-power attenuation was observed from -800 to $-600 \mathrm{~ms}$ and from -650 to $-550 \mathrm{~ms}$, for the After- $R$ and rotation-strategy trials, respectively. Beta-power profiles for the After- $R$ and the rotationstrategy trials ( $R-S 1, R-S 2$, and $R-S 3$ pooled) did not differ significantly from each other.

We complemented the preceding results by one-tailed preplanned pairwise tests (FDR-corrected for multiple comparisons along the time axis, without Bonferroni correction for multiple pairwise contrasts) contrasting each category of trials, $R-S 1, R-S 2$,
$R-S 3$, and After- $R$ trials with the Baseline trials (mean \pm SE number of trials per category: $38 \pm 2.56$ ). These comparisons revealed significant beta-power attenuations for the $R-S 1, R-S 2$, and After- $R$ trials, whereas no significant difference was found between the R-S3 and the Baseline trials (Fig. 6B). The observed beta-power attenuations were more pronounced for the $R-S 1$ and After- $R$ trials (with significant effects from -850 to $-50 \mathrm{~ms}$ and from -1050 to $-500 \mathrm{~ms}$, respectively) than for the $R-S 2$ (significant effects from -750 to $-550 \mathrm{~ms}$ ).

To summarize, as we expected, during movement preparation, different patterns of modulation were observed for the two spatially distinct beta-band activities. Beta-band activity captured by the medial frontal ICs exhibited reduced power when participants applied the explicit re-aiming strategy $(R-S 1-3$ trials) relative to when they did not have to counter a visual rotation (Baseline and After-R trials, which did not differ from each other). 


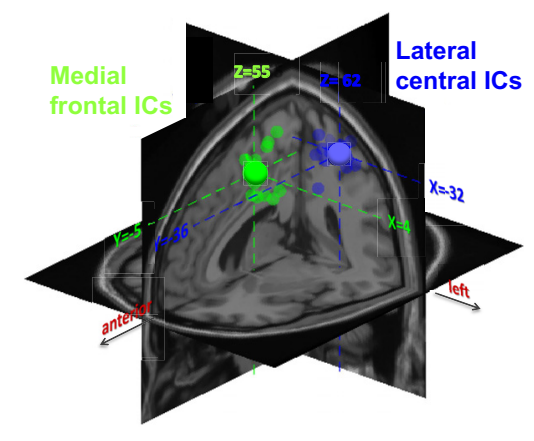

Figure 7. Dipoles for the medial frontal and lateral central ICs. Estimated dipole locations within a three-shell BEM of the MNI standard brain, for the medial (green) and lateral (blue) ICs. Small spheres represent dipoles for each participant. Large spheres represent centroids of the individual dipoles.

Furthermore, this effect was the strongest when participants returned to the deliberate strategy ( $R$-S1 trials) after a series of trials performed without it. In contrast, beta power in the lateral central ICs was reduced for both the strategy trials $(R-S 1-3)$, in which sensory prediction errors were experienced, as well as for the postrotation trials (After-R), relative to the Baseline trials. Interestingly, a gradual decrease of the effect was observed over the strategy trials.

\section{Dipole fitting of the ICs}

For each participant, we computed the equivalent current dipole model for the medial and the lateral central ICs. Figure 7 shows the individual dipole solutions for the two types of ICs together with the corresponding group-averaged dipoles. Dipole centroids were localized to the supplementary motor area (MNI coordinates: $X=4, Y=-5, Z=55)$ for the medial frontal ICs, and to the left somatosensory area (MNI coordinates: $X=-32, Y=$ $-36, Z=62)$ for the lateral central ICs.

\section{Discussion}

Our goal was to determine whether implicit sensorimotor adaptation and deliberate re-aiming are reflected by functionally and spatially distinct beta-band modulations. In previous studies (Torrecillos et al., 2015; Alayrangues et al., 2019), we found that, during the foreperiod, beta-band activity in lateral central regions was modulated in relation to adaptive mechanisms triggered after movement-execution errors were experienced. Based on the nature of our experimental designs, we inferred that these oscillatory responses were related to implicit adaptive processes. In the present study, we used a task in which both implicit sensorimotor remapping and deliberate strategies contributed to task performance. Based on previous findings (Hosaka et al., 2016), we hypothesized that beta-band activity in medial motor cortex would be a potential neural correlate of explicit re-aiming strategy.

We used ICA to separate beta-band activities within medial frontal and lateral regions. During the foreperiod before movement onset, the two spatially distinct beta-band activities exhibited different patterns of modulation, revealing functional differences consistent with our main hypothesis.

\section{Beta-band activity in medial motor cortex reflects strategic re-aiming}

Medial frontal beta power was significantly decreased before movements in which participants deliberately re-aimed toward a location offset from the illuminated target, relative to trials in which they did not have to counter a visual rotation (Baseline and
After- $R$ trials). Our dipole source analysis suggested generators located in the caudal part of the supplementary motor complex (SMC). This finding is in line with previous observations demonstrating the role of the medial frontal cortex in top-down executive control of behavior, specifically when automatic motor responses must be replaced with deliberately controlled actions following a change in the environment. Through electrophysiological recordings in macaque monkeys, Isoda and Hikosaka (2007) have provided anatomical and physiological accounts for the controlled process with which organisms are able to dynamically switch actions in a constantly changing environment. Using an action-switching paradigm, these authors showed single-cell activity specifically preceding motor response switches in the presupplementary motor area (pre-SMA), and improved switching behavior following microstimulation of the same region. Furthermore, by varying the timing of the stimulation, they demonstrated that the pre-SMA enables action switching by first suppressing an automatic inappropriate motor response and then facilitating a controlled desirable movement.

Interestingly, we observed more sustained beta-power attenuation before the first rotation-strategy trial $(R-S 1)$ than before the following ones $(R-S 2, R-S 3)$. This pattern fits with previous observations by Hosaka et al. (2016). The hypothesis of these authors was that the decrease in beta power in SMC during the early delay contributes to the updating of action plans. They analyzed the temporal modulation of beta activity in the local field potentials of the pre-SMA and SMA-proper in monkeys performing a motor task in which they were required to internally maintain a series of movements and update it in response to a visual instruction. Beta power was significantly suppressed during the early delay period of updating trials (in which the sequence of movements had to be changed) in the two medial motor areas. In the following maintenance trials, beta power tended to increase as the new action plan was internalized. Hosaka et al. (2016) proposed an interpretation in terms of dynamic cell assemblies, according to which enhancement of beta power may play a role in protecting preorganized cell assemblies while suppression of beta power may afford permission to update these cell assemblies. In light of these findings, the decrease in beta power observed in the strategic re-aiming trials may be attributable to the inhibition of the motor response automatically triggered by the illuminated target, and the facilitation of the deliberate re-aiming movement.

Alternatively, beta-band desynchronization may have been greater in the re-aiming condition due to increased task complexity, independent of the specific nature of the implicated neural mechanisms. The SMC is classically assumed to be preferentially recruited in the generation of internally driven rather than sensory-guided movements (Goldberg, 1985; Okano and Tanji, 1987; Passingham, 1987; Roland, 1987; Mushiake et al., 1991). According to Nachev et al. (2008), the critical aspect that differentiates these two types of motor actions is their intrinsic level of complexity, rather than in the internal versus external nature of their sources. Self-generated actions involve, for instance, integrating past experience, and are likely to be associated with greater complexity than when externally specified.

\section{Lateral central beta-band activity relates to implicit sensorimotor remapping}

In previous studies (Torrecillos et al., 2015; Alayrangues et al., 2019), we found that the foreperiod beta-band activity in lateral central regions was modulated in relation to adaptive responses that were irrelevant to the outcome of the task and were therefore 
most likely a reflection of implicit processes. Here, for the postrotation and strategic trials, we observed strikingly similar profiles of modulation for the beta-band activity captured by the lateral central ICs (see Torrecillos et al., 2015, their Fig. 9B; Alayrangues et al., 2019, their Fig. 7-8).

One possible explanation for the observed beta power modulations may be that they reflect changes in efferent mechanisms within the motor cortex, elicited by implicit adaptation and behaviorally manifested in the postrotation $(A f t e r-R)$ trials. Indeed, premovement beta desynchronization over motor regions has been interpreted as reflecting their level of activation (Pfurtscheller and Berghold, 1989; Sanes and Donoghue, 1993; Murthy and Fetz, 1996; Schnitzler et al., 1997). Interestingly, Grent-'tJong et al. (2015) reported a reduction in premovement beta power during visuomotor adaptation compared with baseline trials before adaptation, indicating increased neural activity associated with the learned movement directions. Furthermore, their findings would be compatible with the local nature of neural changes related to the learning and retention of new visuomotor mappings within the primary motor cortex (Paz et al., 2003).

This first interpretation is, however, difficult to conciliate with some of our observations. In the present experiment, beta power was significantly decreased before the strategy trials, and this effect gradually attenuated over the three strategy trials. However, no analogous pattern was observed in the behavioral data; movement kinematics did not differ significantly across the strategy trials. Such uncoupling between behavioral and electrophysiological responses is reminiscent of data reported in a recent study from our group (Alayrangues et al., 2019), in which trial-to-trial adaptive motor-command update and premovement lateralized beta-band modulation exhibited different patterns. Specifically, in this study, participants had to perform a so-called Cooperative task in which they had to control a single cursor with both arms to reach a single visual target. In catch trials, a mechanical perturbation was applied pseudo-randomly to the left $(10 \%)$ or the right arm (10\%). While behavioral adaptive responses were visible for both arms, significant beta power modulation was observed only over the right hemisphere regardless of the side of the perturbation. This finding revealed that the lateralized foreperiod beta activity did not reflect efferent processes shaping the muscle activation pattern. Together, these data are difficult to conciliate with a purely "motor" explanation.

An alternative interpretation for the gradual decrease of this effect across the three strategy trials is that it resulted from a progressive reduction of the sensory prediction error through trial-by-trial implicit remapping. This possibility is, however, invalidated by the large beta-band suppression observed in the postrotation trials $($ After-R), which were not preceded by a larger sensory prediction error than the strategic trials $(R-S 3)$. Therefore, yet another speculation may be considered, which better fits our data as well as prevailing motor control theories (Shadmehr and Krakauer, 2008). According to these theories, the cerebellum implements and updates predictive internal models based on sensory prediction errors, whereas the parietal cortex combines these predictions with actual sensory information to estimate the current state of the body and the world (Shadmehr and Krakauer, 2008). Movement planning is based on this estimate, which is statistically optimal (in the Bayesian sense) in that it is obtained by combining predictions (prior beliefs) and sensory input (new evidence) according to their respective uncertainties. Any change in the body and/or the world renders internal model predictions more uncertain and, as a consequence, increases the weight allocated to peripheral sensory input. Within this framework, the gradual decrease in beta-power attenuation across the strategy trials would mirror a gradual decrease in the uncertainty of the sensory prediction as the visual condition remains transiently (for four trials) constant, with as a counterpart a decreased weighting of the sensory input. The rebound of the effect for the postrotation trial would be induced by the anticipated change in the visual condition, making sensory prediction uncertain again. Interestingly, beta-band oscillations have been shown to be suppressed by somatosensory demands, both in anticipation of (Bauer et al., 2006; Jones et al., 2010; van Ede et al., 2011) and during sensory processing (Chatrian et al., 1959; Cheyne et al., 2003; van Ede et al., 2011). Thus, modulations in lateralized beta-band activity may reflect sensory integration processes involved in implicit sensorimotor adaptation (see also Palmer et al., 2019). Furthermore, this view fits with the results of our dipole source analysis, consistent with generators within somatosensory regions.

Hence, the pattern of premovement beta-power modulation that we observed for the strategy and the postrotation trials may reflect an increase in somatosensory processing implicitly triggered in response to a change in the visual condition, experienced through sensory prediction errors or anticipated based on a contextual cue and requiring an update of the internal model sensory predictions.

\section{Implications for implicit and explicit forms of adaptation}

While there is consensus that both implicit sensorimotor adaptation and deliberate cognitive strategies contribute to motor adaptation, a central issue pertains to their interplay. Mazzoni and Krakauer (2006) offered an especially striking illustration of the way the implicit process can interfere with the explicit goal of a motor task. In their experiment, participants were initially able to counter the visual rotation by applying the re-aiming strategy. However, their performance gradually deteriorated as they implicitly adapted to the rotation. According to the authors, this demonstrated that the two processes operate independently, likely implemented through distinct circuits. Taylor and Ivry (2011) added to this finding in a critical way by revealing that, with extended training, participants eventually modify their reaiming strategy so as to offset the detrimental effect (drift) of the implicit process and proposed a model in which performance ultimately stabilizes through a dynamic synergy between the two learning processes. Our behavioral and electrophysiological data fit with these previous observations. Specifically, the functional distinction we draw between beta-band activities captured by the medial and lateral central ICs provides evidence in support of physiologically dissociable processes. An important question for further research, which the present work cannot speak to, is whether the two adaptation processes act independently or not.

\section{References}

Aguera PE, Jerbi K, Caclin A, Bertrand O (2011) ELAN: a software package for analysis and visualization of MEG, EEG, and LFP signals. Comput Intell Neurosci 2011:158970.

Alayrangues J, Torrecillos F, Jahani A, Malfait N (2019) Error-related modulations of the sensorimotor post-movement and foreperiod beta-band activities arise from distinct neural substrates and do not reflect efferent signal processing. Neuroimage 184:10-24.

Baker SN (2007) Oscillatory interactions between sensorimotor cortex and the periphery. Curr Opin Neurobiol 17:649-655.

Bauer M, Oostenveld R, Peeters M, Fries P (2006) Tactile spatial attention enhances gamma-band activity in somatosensory cortex and reduces lowfrequency activity in parieto-occipital areas. J Neurosci 26:490-501.

Chatrian GE, Petersen MC, Lazarte JA (1959) The blocking of the rolandic 
wicket rhythm and some central changes related to movement. Electroencephalogr Clin Neurophysiol 11:497-510.

Cheyne D, Gaetz W, Garnero L, Lachaux JP, Ducorps A, Schwartz D, Varela FJ (2003) Neuromagnetic imaging of cortical oscillations accompanying tactile stimulation. Brain Res Cogn Brain Res 17:599-611.

Delorme A, Sejnowski T, Makeig S (2007) Enhanced detection of artifacts in EEG data using higher-order statistics and independent component analysis. Neuroimage 34:1443-1449.

Doyon J, Penhune V, Ungerleider LG (2003) Distinct contribution of the cortico-striatal and cortico-cerebellar systems to motor skill learning. Neuropsychologia 41:252-262.

Engel AK, Fries P (2010) Beta-band oscillations: signalling the status quo? Curr Opin Neurobiol 20:156-165.

Goldberg G (1985) Supplementary motor area structure and function: review and hypotheses. Behav Brain Sci 8:567-616.

Grent-'t-Jong T, Oostenveld R, Medendorp WP, Praamstra P (2015) Separating visual and motor components of motor cortex activation for multiple reach targets: a visuomotor adaptation study. J Neurosci 35: $15135-15144$

Hosaka R, Nakajima T, Aihara K, Yamaguchi Y, Mushiake H (2016) The suppression of beta oscillations in the primate supplementary motor complex reflects a volatile state during the updating of action sequences. Cereb Cortex 26:3442-3452.

Isoda M, Hikosaka O (2007) Switching from automatic to controlled action by monkey medial frontal cortex. Nat Neurosci 10:240-248.

Jenkinson N, Brown P (2011) New insights into the relationship between dopamine, beta oscillations and motor function. Trends Neurosci 34: 611-618.

Jones SR, Kerr CE, Wan Q, Pritchett DL, Hämäläinen M, Moore CI (2010) Cued spatial attention drives functionally relevant modulation of the mu rhythm in primary somatosensory cortex. J Neurosci 30:13760-13765.

Jordan MI, Rumelhart DE (1992) Forward models: supervised learning with a distal teacher. Cogn Sci 16:307-354.

Kilavik BE, Zaepffel M, Brovelli A, MacKay WA, Riehle A (2013) The ups and downs of $\beta$ oscillations in sensorimotor cortex. Exp Neurol 245: $15-26$.

Little S, Pogosyan A, Neal S, Zavala B, Zrinzo L, Hariz M, Foltynie T, Limousin P, Ashkan K, FitzGerald J, Green AL, Aziz TZ, Brown P (2013) Adaptive deep brain stimulation in advanced Parkinson disease. Ann Neurol 74:449-457.

Makeig S, Jung TP, Bell AJ, Ghahremani D, Sejnowski TJ (1997) Blind separation of auditory event related brain responses into independent components. Proc Natl Acad Sci U S A 94:10979-10984.

Malfait N, Ostry DJ (2004) Is interlimb transfer of force-field adaptation a cognitive response to the sudden introduction of load? J Neurosci 37:8084-8089.

Mazzoni P, Krakauer JW (2006) An implicit plan overrides an explicit strategy during visuomotor adaptation. J Neurosci 26:3642-3645.

Meidahl AC, Moll CKE, van Wijk BCM, Gulberti A, Tinkhauser G, Westphal M, Engel AK, Hamel W, Brown P, Sharott A (2019) Synchronised spiking activity underlies phase amplitude coupling in the subthalamic nucleus of Parkinson's disease patients. Neurobiol Dis 127:101-113.

Murthy VN, Fetz EE (1996) Oscillatory activity in sensorimotor cortex of awake monkeys: synchronization of local field potentials and relation to behavior. J Neurophysiol 76:3949-3967.

Mushiake H, Inase M, Tanji J (1991) Neuronal activity in the primate premotor, supplementary and precentral motor cortex during visually guided and internally determined sequential movements. J Neurophysiol 66:705-718.

Nachev P, Kennard C, Husain M (2008) Functional role of the supplementary and pre-supplementary motor areas. Nat Rev Neurosci 9:856-869.

Nakamura K, Sakai K, Hikosaka O (1998) Neuronal activity in medial frontal cortex during learning of sequential procedures. J Neurophysiol 80:2671-2687.
Okano K, Tanji J (1987) Neuronal activities in primate motor fields of the agranular frontal cortex preceding visually triggered and self-paced movement. Exp Brain Res 66:155-166.

Oldfield RC (1971) The assessment and analysis of handedness: the Edinburgh Inventory. Neuropsychologia 9:97-113.

Oostenveld R, Fries P, Maris E, Schoffelen JM (2011) FieldTrip: open source software for advanced analysis of MEG, EEG and invasive electrophysiological data. Comput Intell Neurosci 2011:156869.

Palmer CE, Auksztulewicz R, Ondobaka S, Kilner JM (2019) Sensorimotor beta power reflects the precision-weighting afforded to sensory prediction errors. Neuroimage 200:59-71.

Passingham RE (1987) Two cortical systems for directing movement. Ciba Found Symp 132:151-164.

Paz R, Boraud T, Natan C, Bergman H, Vaadia E (2003) Preparatory activity in motor cortex reflects learning of local visuomotor skills. Nat Neurosci 6:882-890.

Pfurtscheller G, Berghold A (1989) Patterns of cortical activation during planning of voluntary movement. Electroencephalogr Clin Neurophysiol 72:250-258.

Picard N, Strick PL (1996) Motor areas of the medial wall: a review of their location and functional activation. Cereb Cortex 6:342-353.

Redding GM, Wallace B (1996) Adaptive spatial alignment and strategic perceptual-motor control. J Exp Psychol Hum Percept Perform 22: 379-394.

Roland PE (1987) Metabolic mapping of sensorimotor integration in the human brain. Ciba Found Symp 132:251-268.

Rushworth MF, Walton ME, Kennerley SW, Bannerman DM (2004) Action sets and decisions in the medial frontal cortex. Trends Cogn Sci 8:410-417.

Saleh M, Reimer J, Penn R, Ojakangas CL, Hatsopoulos NG (2010) Fast and slow oscillations in human primary motor cortex predict oncoming behaviorally relevant cues. Neuron 65:461-471.

Sanes JN, Donoghue JP (1993) Oscillations in local field potentials of the primate motor cortex during voluntary movement. Proc Natl Acad Sci U S A 90:4470-4474.

Schnitzler A, Salenius S, Salmelin R, Jousmäki V, Hari R (1997) Involvement of primary motor cortex in motor imagery: a neuromagnetic study. Neuroimage 6:201-208.

Shadmehr R, Krakauer JW (2008) A computational neuroanatomy for motor control. Exp Brain Res 185:359-381.

Tan H, Jenkinson N, Brown P (2014) Dynamic neural correlates of motor error monitoring and adaptation during trial-to-trial learning. J Neurosci 34:5678-5688.

Taylor JA, Ivry RB (2011) Flexible cognitive strategies during motor learning. Plos Comput Biol 7:e1001096.

Taylor JA, Krakauer JW, Ivry RB (2014) Explicit and implicit contributions to learning in a sensorimotor adaptation task. J Neurosci 34:3023-3032.

Tinkhauser G, Pogosyan A, Little S, Beudel M, Herz DM, Tan H, Brown P (2017) The modulatory effect of adaptive deep brain stimulation on beta bursts in Parkinson's disease. Brain 140:1053-1067.

Torrecillos F, Alayrangues J, Kilavik BE, Malfait N (2015) Distinct modulations in sensorimotor postmovement and foreperiod beta-band activities related to error salience processing and sensorimotor adaptation. J Neurosci 35:12753-12765.

van Ede F, de Lange F, Jensen O, Maris E (2011) Orienting attention to an upcoming tactile event involves a spatially and temporally specific modulation of sensorimotor alpha- and beta-band oscillations. J Neurosci 31:2016-2024.

von Helmholtz HL (1867) Handbuch der physiologischen Optik Leipzig: L. Voss. Reprinted, with extensive commentary in (Gullstrand A, von Kries J, Nagel W, eds), Handbuch der physiologischen Optik (Ed 3). Leipzig: L. Voss, 1910 